\title{
EXTENSIONS OF SEMIGROUPS
}

\author{
BY \\ A. H. CLIFFORD
}

By the term semigroup we shall mean a system consisting of a class $\Sigma$ of elements, $a, b, c, \cdots$ in which there is defined an associative binary operation: $a(b c)=(a b) c$. An ideal of $\Sigma$ is a subset $S$ of $\Sigma$ such that if $a$ is in $\Sigma$ and $b$ is in $S$, then both products $a b$ and $b a$ are in $S$. Rees $\left({ }^{1}\right)$ defines the difference semigroup $T=\Sigma-S$ to be essentially that obtained by collapsing $S$ into a single zero element 0 , while the remaining elements of $\Sigma$ retain their identity. Thus the $T$-product of two nonzero elements is defined to be 0 if their $\Sigma$-product lies in $S$, and otherwise to be the same as defined in $\Sigma\left({ }^{2}\right)$. As in the Schreier theory of group extensions, let us consider the problem of constructing, for given semigroup $S$ and given semigroup $T$ with zero, every possible semigroup $\Sigma$ containing $S$ as an ideal, such that $\Sigma-S$ is isomorphic with $T$. Such a $\Sigma$ will be called an extension of $S$ by $T$.

If $S$ has a two-sided identity element, every extension of $S$ by $T$ can be obtained by means of a "ramified homomorphism" of $T$ into $S$ (Theorem 2). If $S$ satisfies the mild "Condition A," stated in $\S 1$, every extension of $S$ by $T$ can be obtained by means of a pair of "linked" ramified homomorphisms of $T$ into the semigroups of left and right translations of $S$ (Theorem 3 ). Condition A is satisfied if $S$ is what Rees (loc. cit. p. 393) calls a completely simple semigroup without zero. This case is of interest because $S$ is then the "Suschkewitsch kernel" of $\Sigma$, originally described by Suschkewitsch $\left(^{3}\right)$ for finite semigroups, shown by Rees (loc. cit. p. 392) to be the intersection of all the ideals of $\Sigma$, and further studied by $\operatorname{Schwarz}\left({ }^{4}\right)$ and the author $\left(^{5}\right)$. In a previous paper $\left(^{6}\right)$ the author gave an extension theorem for such an $S$ by a semigroup $T$ having no divisors of zero. Theorem 5 below extends this to arbitrary $T$.

Presented to the Society, October 29, 1949; received by the editors January 17, 1949 and, in revised form, August 1, 1949.

( $\left.{ }^{1}\right)$ D. Rees, On semi-groups, Proc. Cambridge Philos. Soc. vol. 36 (1940) pp. 387-400. The definition of difference semigroup is on p. 389,

(2) For the analogue of this definition in ring theory, let $\Sigma$ be the set of basis elements of an algebra over some field. Then the elements of $S$ constitute a basis of an invariant subalgebra. The elements of $\Sigma$ not in $S$ form a basis of the difference algebra, and multiply therein as described above.

(3) A. Suschkewitsch, Über die endlichen Gruppen ohne das Gesetz der eindeutigen Umkehrbarkeit, Math. Ann. vol. 99 (1928) pp. 30-50.

(4) Stefan Schwarz, Zur Theorie der Halbgruppen (Slovakian, German Summary), Sborník prác Prírodovedeckej fakulty Slovenskej univerzity v Bratislave, No. 6, 1943, 64 pp. See Mathematical Reviews vol. 10 (1949) p. 12.

(5) A. H. Clifford, Semigroups containing minimal ideals, Amer. J. Math. vol. 70 (1948) pp. 521-526.

(') A. H. Clifford, Semigroups admitting relative inverses, Ann. of Math. vol. 42 (1941) pp. 1037-1049. The reference is to Theorem 4 on p. 1047. This paper will be referred to as "Rel. Inv." 
1. Notation and preliminary considerations. In the sequel, the semigroup $S$ will sometimes be subject to one of the following conditions.

Condition A. If $a s=b s$ and $s a=s b$ for all $s$ in $S$ then $a=b$.

Condition B. To each $a$ in $S$ correspond $u$ and $v$ in $S$ such that $u a=a v=a$.

Lemma 1. Condition A is a consequence of Condition B.

Proof. Assume Condition B and suppose that $a$ and $b$ are elements of $S$ such that $a s=b s$ and $s a=s b$ for all $s$ in $S$. By Condition B there exist $u$ and $v$ in $S$ such that $u a=a$ and $b v=b$. Then

$$
a=u a=u b=u b v=a v=b v=b .
$$

Every completely simple semigroup $S$ satisfies Condition B (and hence also A). For one of the defining conditions thereof (Rees, loc. cit. p. 393, condition (ii)) is simply (B) with the added requirement that $u$ and $v$ be idempotent.

To save repetition, we shall adhere throughout the paper to the following notation. $S$ will denote any semigroup, possibly satisfying Condition A or B, and in $\$ 5$ it will be a completely simple semigroup without zero. $T$ will denote any semigroup with zero element 0 , having no elements in common with $S$. $T^{*}$ will denote the set of nonzero elements of $T$. Except in $\$ 5$, the small letters $a, b, c, e, s, t, u, v$ will always denote elements of $S$. The capitals $A, B, C$ will always denote elements of $T^{*}$, even in $\S 5$. Expressions like "for all $A, B$ in $T^{*}$ and all $s$ in $S$ " will be omitted unless necessary for emphasis.

$\Sigma$ will denote the class sum of $S$ and $T^{*}$. The construction of an extension of $S$ by $T$ requires firstly the definition of a binary operation $\circ$ in $\Sigma$ subject to the conditions (P 1-4):

$$
A \circ B \begin{cases}=A B & \text { if } \quad A B \neq 0, \\ \in S & \text { if } \quad A B=0 .\end{cases}
$$

$$
A \circ s \in S \quad \text { (P 3) } \quad s \circ A \in S
$$

(P 4) $\quad s \circ t=s t$.

The resulting system $\Sigma(0)$ will be an extension of $S$ by $T$ if and only if the associative law holds. This breaks down into $2^{3}=8$ cases according to whether each of the three factors is in $S$ or in $T^{*}$. The case $S S S$ where all three factors are in $S$ is naturally immediate from (P 4) and associativity in $S$. The most troublesome case in practise is $T^{*} T^{*} T^{*}$, namely $(A \circ B) \circ C$ $=A \circ(B \circ C)$. If $A B C \neq 0$ this reduces to associativity in $T$ by (P 1$)$, but if $A B C=0$ we have four subcases according to whether $A B$ and $B C$ are $=0$ or $\neq 0$.

Theorem 1. If $S$ satisfies Condition A, the associativity cases $T^{*} S T^{*}$ and $T^{*} T^{*} T^{*}$ are consequences of the remaining ones. 
Proof.

$$
\begin{aligned}
& ((A \circ B) \circ C) \circ s=(A \circ B) \circ(C \circ s) \text { by }\left\{\begin{array}{lll}
T^{*} T^{*} S & \text { if } A B \neq 0, \\
S T^{*} S & \text { if } A B=0 ;
\end{array}\right. \\
& ((A \circ B) \circ C) \circ s=A \circ(B \circ(C \circ s)) \text { by } T^{*} T^{*} S \text {; } \\
& ((A \circ B) \circ C) \circ s=A \circ((B \circ C) \circ s) \text { by } T^{*} T^{*} S \text {; } \\
& ((A \circ B) \circ C) \circ s=(A \circ(B \circ C)) \circ s \text { by }\left\{\begin{array}{lll}
T^{*} T^{*} S & \text { if } & B C \neq 0, \\
T^{*} S S & \text { if } & B C=0 .
\end{array}\right.
\end{aligned}
$$

Similarly we can show that

$$
s \circ((A \circ B) \circ C)=s \circ(A \circ(B \circ C))
$$

without using the cases $T^{*} S T^{*}$ or $T^{*} T^{*} T^{*}$. From Condition A we conclude $(A \circ B) \circ C=\left(A \circ(B \circ C)\right.$. The proof of case $T^{*} S T^{*}$ is exactly the same as above, if we replace $B$ by an element $b$ of $S$.

2. Extensions defined by ramified homomorphisms. By a ramified homomorphism of $T$ into $S$ we shall mean a single-valued mapping $A \rightarrow \bar{A}$ of $T^{*}$ into $S$ such that if $A B \neq 0$ then $\overline{A B}=\bar{A} \bar{B}$. The reason for the term "ramified" is that, in a sense, the zero element of $T$ corresponds to a whole set of elements of $S$, namely that of all products $\bar{A} \bar{B}$ with $A B=0$.

Theorem 2. A ramified homomorphism $A \rightarrow \bar{A}$ of $T$ into $S$ determines an extension $\Sigma(\circ)$ of $S$ by $T$ as follows:

$$
A \circ B=\left\{\begin{array}{lll}
A B & \text { if } & A B \neq 0, \\
\bar{A} \bar{B} & \text { if } & A B=0 ;
\end{array}\right.
$$

$A \circ s=\bar{A} s$

(M 3)

$$
s \circ A=s \bar{A}
$$

(M 4) $\quad s \circ t=s t$.

If $S$ has a two-sided identity element, then every extension of $S$ by $T$ is found in this fashion.

Proof. The proof of the first half of the theorem is simply a routine verification of the associative law, so we shall give only the most troublesome case $T^{*} T^{*} T^{*}$. If $A B C=0$, then

$$
\begin{aligned}
& (A \circ B) \circ C=\left\{\begin{array}{lll}
(A B) \circ C=(\overline{A B}) \bar{C}=(\bar{A} \bar{B}) \bar{C} & \text { if } & A B \neq 0, \\
(\bar{A} \bar{B}) \circ C=(\bar{A} \bar{B}) \bar{C} & \text { if } & A B=0 ;
\end{array}\right. \\
& A \circ(B \circ C)=\left\{\begin{array}{lll}
A \circ(B C)=\bar{A}(\overline{B C})=\bar{A}(\bar{B} \bar{C}) & \text { if } & B C \neq 0, \\
A \circ(\bar{B} \bar{C})=\bar{A}(\bar{B} \bar{C}) & \text { if } & B C=0 .
\end{array}\right.
\end{aligned}
$$

In all four subcases, associativity follows from that in $S$.

Proceeding to the converse, let $e$ be the identity element of $S$. From (P 2-4) and associativity in $\Sigma$ (0) we have 


$$
A \circ e=e(A \circ e)=e \circ(A \circ e)=(e \circ A) \circ e=(e \circ A) e=e \circ A \text {. }
$$

Define $\bar{A}=A \circ e(=e \circ A)$. Then from the above and (P 1),

$$
\begin{aligned}
\bar{A} \bar{B} & =A \circ e \circ B \circ e=A \circ B \circ e \circ e=A \circ B \circ e \\
& = \begin{cases}(A B) \circ e=\overline{A B} & \text { if } A B \neq 0, \\
(A \circ B) e=A \circ B & \text { if } A B=0 .\end{cases}
\end{aligned}
$$

The first of these shows that $A \rightarrow \bar{A}$ is a ramified homomorphism of $T$ into $S$. The second establishes the second part of (M 1). To show (M 2) we have

$$
A \circ s=A \circ e \circ s=\bar{A} \circ s=\bar{A} s
$$

and the proof of (M 3) is dual thereto. (M 4) and the first part of (M 1) do not of course require proof.

The referee raises the pertinent question of how to tell, for given $S$ and $T$, whether an extension of $S$ by $T$ exists, and gives the following example where no extension exists: $S$ the infinite cyclic semigroup $a, a^{2}, \cdots$ and $T$ the multiplicative semigroup of the ring of integers modulo 15. The author is not able to answer this question. At least Theorem 2 shows that an extension of $S$ by $T$ always exists if $S$ contains an idempotent element, which is the case, for example, if $S$ is completely simple.

3. Left and right translations of a semigroup. Let $\Sigma(0)$ be an extension of $S$ by $T$. By associativity in $\Sigma(0)$, the mapping $s \rightarrow A \circ s=\lambda_{A} s$ (for fixed $A$ in $T^{*}$ ) of $S$ into itself has the property $\lambda_{A}(s t)=\left(\lambda_{A} s\right) t$. Any mapping $\lambda$ of $S$ into itself with this property, $\lambda(s t)=(\lambda s) t$, we shall call a left translation of $S$. Similarly $s \rightarrow s \circ A=s \rho_{A}$ is a right translation of $S$, that is, $(s t) \rho_{A}$ $=s\left(t \rho_{A}\right)$.

If $c$ is a fixed element of $S$, the mapping $s \rightarrow c s$ will be called the special left translation induced by $c$, and will be denoted by $\lambda_{c}$. If $S$ has a left identity element $e$ then every left translation of $S$ is special, for $\lambda s=\lambda(e s)=(\lambda e) s=c s$ with $c=\lambda e$.

If $\lambda_{1}$ and $\lambda_{2}$ are left translations of $S$, so also is their product $\lambda_{1} \lambda_{2}$. For

$$
\left(\lambda_{1} \lambda_{2}\right)(s t)=\lambda_{1}\left(\lambda_{2}(s t)\right)=\lambda_{1}\left(\left(\lambda_{2} s\right) t\right)=\left(\lambda_{1}\left(\lambda_{2} s\right)\right) t=\left(\left(\lambda_{1} \lambda_{2}\right) s\right) t .
$$

The identity mapping $s \rightarrow s$ is clearly a left translation. Thus the set $\Lambda$ of all left translations of a semigroup $S$ is a semigroup with two-sided identity element. Since $\lambda_{a} \lambda_{b}=\lambda_{a b}$ the subset $\Lambda_{0}$ of all special left translations is a subsemigroup of $\Lambda$, and the mapping $a \rightarrow \lambda_{a}$ is a homomorphism of $S$ onto $\Lambda_{0}$. (Since $\lambda \lambda_{a}=\lambda_{\lambda a}, \Lambda_{0}$ is in fact a left ideal of $\Lambda$.) If $S$ has a two-sided identity element, then $\Lambda=\Lambda_{0} \cong S$.

Similarly the set $\mathbf{P}$ of all right translations of $S$ is a semigroup containing the subsemigroup (in fact right ideal) $\mathbf{P}_{0}$ of special right translations $\rho_{c}: s \rightarrow s \rho_{c}=s c$ ( $c$ fixed in $S$ ), and $c \rightarrow \rho_{c}$ is a homomorphism of $S$ onto $\mathrm{P}_{0}$.

A product $\lambda_{1} \lambda_{2}$ of left translations means first $\lambda_{2}$, then $\lambda_{1}$, while a product 
$\rho_{1} \rho_{2}$ of right translations means first $\rho_{1}$, then $\rho_{2}$. This will not lead to confusion since we shall avoid writing a product $\lambda \rho$.

Incidentally, it is not always true that every left translation commutes with every right translation, but it is true at least in the following two cases: (1) $S^{2}=S$; (2) $S$ satisfies Condition A, every left translation of $S$ is linked with some right translation of $S$, and vice-versa. We say that $\lambda$ and $\rho$ are linked if

$$
s(\lambda t)=(s \rho) t \quad \text { for all } s, t \in S .
$$

The following lemma will be used only for the more or less incidental Theorem 4.

Lemma 2. Let $S$ satisfy Condition B. If $\lambda_{a}$ and $\rho_{b}$ are linked special left and right translations of $S$, that is, sat $=s b t$ for all $s$ and $t$ in $S$, then there exists $c$ in $S$ such that $\lambda_{c}=\lambda_{a}$ and $\rho_{c}=\rho_{b}$.

Proof. By Condition B (see $\$ 1$ ) there exist $u$ and $v$ in $S$ such that $u a=a$ and $b v=b$. Let $c=a v$. Then $c=u a v=u b v=u b$, and

$$
\begin{aligned}
& c s=u b s=u a s=a s, \text { that is, } \lambda_{c}=\lambda_{a}, \\
& s c=s a v=s b v=s b, \text { that is, } \rho_{c}=\rho_{b} .
\end{aligned}
$$

4. Extensions defined by translations. Equations (P 1-4) may be written in the equivalent form

$$
A \circ B= \begin{cases}A B & \text { if } \quad A B \neq 0, \\ {[A, B]} & \text { if } \quad A B=0 ;\end{cases}
$$

(N 2) $A \circ s=\lambda_{A} s$;

(N 3) $\quad s \circ A=s \rho_{A}$;

(N 4) $\quad s \circ t=s t$.

Here $[A, B]$ denotes what we shall call a ramification set of $T^{*}$ in $S$, namely a function defined for pairs $A, B$ of elements of $T^{*}$ such that $A B=0$, and having values in $S . \lambda_{A}$ and $\rho_{A}$ denote, for each fixed $A$ in $T^{*}$, single-valued mappings of $S$ into itself.

Case $T^{*} S S\left[S S T^{*}\right]$ of the associative law is equivalent to the assertion that $\lambda_{A}\left[\rho_{A}\right]$ is a left [right] translation of $S$ (see $\S 3$ ). Cases $T^{*} T^{*} S, S T^{*} T^{*}$, and $S T^{*} S$ are equivalent respectively to the following conditions on the functions $\lambda_{A}, \rho_{A}$, and $[A, B]$ :

$$
\begin{aligned}
\lambda_{A} \lambda_{B} & = \begin{cases}\lambda_{A B} & \text { if } A B \neq 0, \\
\lambda_{[A, B]} & \text { if } A B=0 .\end{cases} \\
\rho_{A} \rho_{B} & =\left\{\begin{array}{lll}
\rho_{A B} & \text { if } A B \neq 0, \\
\rho_{[A, B]} & \text { if } A B=0 .
\end{array}\right. \\
s\left(\lambda_{A} t\right) & =\left(s \rho_{A}\right) t, \quad \text { that is, } \lambda_{A} \text { and } \rho_{A} \text { are linked. }
\end{aligned}
$$

If $S$ satisfies Condition A, Theorem 1 assures us that the remaining two cases, 
$T^{*} S T^{*}$ and $T^{*} T^{*} T^{*}$, are consequences of the foregoing. Hence we have the following restatement of Theorem 1.

Theorem 3. Let $S$ satisfy Condition A. Then every extension $\Sigma(0)$ of $S$ by $T$ is found as follows. Let $A \rightarrow \lambda_{A}$ and $A \rightarrow \rho_{A}$ be mappings of $T^{*}$ into the semigroups $\Lambda$ and $\mathrm{P}$ of left and right translations of $S$, respectively, and let $[A, B]$ be a ramification set of $T^{*}$ in $S$, such that Conditions (C 1-3) are satisfied. Then the class sum $\Sigma$ of $S$ and $T^{*}$ becomes an extension of $S$ by $T$ if product 0 therein is defined by the equations (N 1-4).

We may express this theorem in the following way. If $S$ satisfies Condition A, then every extension of $S$ by $T$ is obtained by means of linked left and right ramified representations of $T^{*}$ by left and right translations of $S$, having a ramification set in common. Suppose now that $A \rightarrow \lambda_{A}$ and $A \rightarrow \rho_{A}$ are linked left and right representations of $T^{*}$ by left and right translations of $S$, but possibly having different ramification sets $[A, B]_{1}$ and $[A, B]_{2}$ respectively.

THEOREM 4. If $S$ satisfies Condition $\mathrm{B}$ there exists a unique ramification set $[A, B]_{3}$ common to $\lambda_{A}$ and $\rho_{A}$.

Proof. By (C 3$)$ the translations $\lambda_{[A, B]_{1}}$ and $\rho_{[A, B]_{2}}$ are linked. By Lemma 2 there exists $[A, B]_{3}$ such that $\lambda_{[A, B]_{1}}=\lambda_{[A, B]_{3}}$ and $\rho_{[A, B]_{2}}=\rho_{[A, B]_{3}}$. The unicity of $[A, B]_{3}$ follows from Condition $\mathrm{A}$, which holds by Lemma 1 .

5. Extensions of a completely simple semigroup without zero. Throughout this section $S$ will denote a completely simple semigroup without zero, represented (Rees, loc. cit. p. 399) as a $J \times K$ matrix semigroup over a group $G^{*}$ with defining matrix $P=\left(p_{k i}\right)$. As in Rel. Inv. $\left({ }^{6}\right)$, the elements of $S$ will be written $(a ; i, \kappa), a \in G^{*}, i \in J, \kappa \in K$, and the product of two such is defined as follows:

$$
(a ; i, \kappa) P(b ; j, \lambda)=\left(a p_{\kappa} b ; i, \lambda\right) .
$$

Let $G$ be $G^{*}$ with a zero adjoined. Then we may think of $(a ; i, \kappa)$ as a matrix with $a$ in the $i$ th row and $\kappa$ th column, and zeros elsewhere. The above product law is then the usual matrix product, but with a constant "sandwich" matrix $P$.

We shall make the following changes in the notation used in $\$ 4$ of Rel. Inv. $S$ will be replaced by $\Sigma, S_{\alpha}$ by $T^{*}, S_{\beta}$ by $S, G$ by $G^{*}$, and product in $\Sigma$ will be denoted by 0 . As in Rel. Inv., we shall assume that $P$ is normalized: $p_{k 1}=p_{1 i}$ $=e$ for all $i \in J, \kappa \in K, e$ being the identity element of $G^{*}$.

TheOREM 5. An extension $\Sigma(0)$ of $S$ by $T$ determines the following three things:

(1) a ramified left representation of $T$ by mappings of the index class $J$ into itself, that is, to each $A$ in $T^{*}$ corresponds a mapping $i \rightarrow A i$ such that

$$
A(B i)= \begin{cases}(A B) i & \text { if } A B \neq 0, \\ i_{A, B} & \text { if } A B=0,\end{cases}
$$


where $i_{A, B}$ is an element of $J$ depending on $A$ and $B$ but not on $i$;

(2) a ramified right representation of $T$ by mappings of the index class $K$ into itself, that is, to each $A$ in $T^{*}$ corresponds a mapping $\kappa \rightarrow \kappa A$ such that

$$
(\kappa A) B= \begin{cases}\kappa(A B) & \text { if } A B \neq 0, \\ \kappa_{A, B} & \text { if } A B=0,\end{cases}
$$

where $\kappa_{A, B}$ is an element of $K$ depending on $A$ and $B$ but not on $\kappa$;

(3) a mapping $A \rightarrow A \phi$ of $T^{*}$ into the structure group $G^{*}$ of $S$ satisfying the two conditions

$$
\begin{gathered}
p_{k, A 1}(A \phi) p_{k A, i}=p_{k, A i}(A \phi) p_{1 A, i}, \\
(A B) \phi=(A \phi) p_{1 A, B 1}(B \phi) \text { if } A B \neq 0 .
\end{gathered}
$$

The connection between the mappings $i \rightarrow A i, \kappa \rightarrow \kappa A, A \rightarrow A \phi$ and product $\circ$ in $\Sigma$ is given by $(Q 1-4)$ :

$$
\begin{array}{rlr}
\text { (Q 1) } & A \circ B & = \begin{cases}A B & \text { if } A B \neq 0, \\
\left(A \phi \cdot p_{1 A, B 1} \cdot B \phi ; i_{A, B}, \kappa_{A, B}\right) & \text { if } A B=0 .\end{cases} \\
\text { (Q 2) } & A \circ(a ; i, \kappa) & =\left(A \phi \cdot p_{1 A, i} a ; A i, \kappa\right) . \\
\text { (Q 3) } & (a ; i, \kappa) \circ A & =\left(a p_{\kappa, A 1} \cdot A \phi ; i, \kappa A\right) . \\
\text { (Q 4) } \quad(a ; i, \kappa) \circ(b ; j, \lambda) & =\left(a p_{\times} ; b ; i, \lambda\right) .
\end{array}
$$

Conversely, if we are given mappings $i \rightarrow A i, \kappa \rightarrow \kappa A, A \rightarrow A \phi$ as described in (1), (2), (3), and define product $\circ$ in the class sum $\Sigma$ of $S$ and $T^{*}$ by (Q 1-4), then $\Sigma(0)$ is an extension of $S$ by $T$.

Proof. All equations in $\$ 4$ of Rel. Inv. remain valid so long as we assume that the product of two elements of $T^{*}$ occurring therein are not equal to 0 . Hence the first part of equations (5.1) and (5.2) are already established by (4.3). Likewise (5.3) is just (4.7), while (5.4) is just (4.8) with the restriction that $A B \neq 0$. ( $Q 2)$ and $(Q 3)$ are the same as (4.9) and (4.10). Hence all we need to show is the second parts of (5.1), (5.2), and (Q 1$)$.

By a double application of $(Q 2)$ we have

$$
A \circ(B \circ(a ; i, \kappa))=\left(A \phi \cdot p_{1 A, B i} \cdot B \phi \cdot p_{1 B, i} \cdot a ; A(B i), \kappa\right) \text {. }
$$

Let the ramification set determined by $\Sigma(0)$ be

$$
[A, B]=\left(g_{A, B} ; i_{A, B}, \kappa_{A, B}\right) .
$$

If $A B=0$ then $A \circ B=[A, B]$ and hence

$$
\begin{aligned}
(A \circ B) \circ(a ; i, \kappa) & =\left(g_{A, B} ; i_{A, B}, \kappa_{A, B}\right) P(a ; i, \kappa) \\
& =\left(g_{A, B} p_{\boldsymbol{\alpha}_{A, B}} a ; i_{A, B}, \kappa\right) .
\end{aligned}
$$

Equating the $J$-component of this and (5.5) we obtain the second part of 
(5.1); the proof of (5.2) is dual. Equating the $G^{*}$-component, setting $i=1$, and using the normalization of $P$, we obtain

$$
g_{A, B}=A \phi \cdot p_{1 A, B 1} \cdot B \phi .
$$

This and (5.6) give (Q 1).

To prove the converse, we remark that all cases of the associative law for $\circ$ have been established in Rel. Inv. except when adjacent factors in $T^{*}$ have vanishing product. Theorem 1 dispenses with the unpleasant case $T^{*} T^{*} T^{*}$, since $(\$ 1) S$ satisfies Condition A. This leaves only the dual cases $T^{*} T^{*} S$ and $S T^{*} T^{*}$, and naturally we need only show one of them. All that remains, therefore, is to show that

$$
A \circ(B \circ(a ; i, \kappa))=(A \circ B) \circ(a ; i, \kappa)
$$

for the subcase $A B=0$. The left side has been calculated in (5.5). The right side has been calculated in (5.7) with $g_{A, B}$ given by (5.8). The $J$-components are equal by (5.1), and their $K$-components are identical. The equality of their $G^{*}$-components will follow if we can show that

$$
p_{1 A, B 1}(B \phi) p_{\boldsymbol{x}_{A, B} i}=p_{1 A, B i}(B \phi) p_{1 B, i} \text {. }
$$

But this follows from (5.3) on replacing $A$ by $B$ and $\kappa$ by $1 A$, and noting from (5.2) that $\kappa_{A, B}=(1 A) B$.

Theorem 5 has been proved without using the notion of a translation of $S$. In spite of this, it may be of some interest to determine the translations of $S$, and to phrase Theorem 3 accordingly.

We shall say that a $J \times J$ matrix $L$ over $G$ is column-restricted if in each column of $L$ there is exactly one nonzero element of $G$. If we multiply an element of $S$ on the left by $L$ we see that the product lies in $S$. From associativity for matrices, this yields a left translation of $S$. But the converse of this is contained implicitly in equation (4.1) of Rel. Inv. Hence the semigroup $\Lambda$ of left translations of $S$ can be represented as the semigroup of all $J \times J$ columnrestricted matrices over $G$. Similarly the semigroup $\mathrm{P}$ of right translations of $S$ can be represented as the semigroup of all $K \times K$ row-restricted matrices over $G$. The following is an immediate consequence of Theorems 3 and 4 .

THEOREM 6. Every extension $\Sigma(0)$ of $S$ by $T$ can be obtained as follows. Let $A \rightarrow L_{A}\left[R_{A}\right]$ be a ramified representation of $T$ by $J \times J$ column-[K$\times K$ row-] restricted matrices, that is,

$$
\begin{aligned}
& L_{A} L_{B}= \begin{cases}L_{A B} & \text { if } A B \neq 0, \\
L_{[A, B]_{1}} & \text { if } A B=0 ;\end{cases} \\
& R_{A} R_{B}= \begin{cases}R_{A B} & \text { if } A B \neq 0, \\
R_{[A, B]_{2}} & \text { if } A B=0 ;\end{cases}
\end{aligned}
$$


where $[A, B]_{1}$ and $[A, B]_{2}$ are ramification sets of $T^{*}$ in $S$. Let these matrices $L_{A}$ and $R_{A}$ be linked by the defining matrix $P$ of $S$ :

$$
P L_{A}=R_{A} P \text {. }
$$

Then there exists a unique ramification set $[A, B]_{3}$ common to $L_{A}$ and $R_{A}$ satisfying (D 1) and (D 2), and the product o defined in $\Sigma=S \cup T^{*}$ by the equations $\left(Q^{\prime} 1-4\right)$ is associative.

$$
\begin{aligned}
A \circ B & = \begin{cases}A B & \text { if } A B \neq 0, \\
{[A, B]_{3}} & \text { if } A B=0 ;\end{cases} \\
A \circ(a ; i, \kappa) & =L_{A}(a ; i, \kappa), \\
(a ; i, \kappa) \circ A & =(a ; i, \kappa) R_{A}, \\
(a ; i, \kappa) \circ(b ; j, \lambda) & =(a ; i, \kappa) P(b ; j, \lambda) .
\end{aligned}
$$

Note added by the referee. Given $S$, consider the semigroup $S^{\prime}$ of all pairs $(\lambda, \rho)$ of linked left and right translations of $S$, that is,

$$
s(\lambda t)=(s \rho) t \quad \text { all } s, t \text { in } S .
$$

If $S$ satisfies Condition A, $S^{\prime}$ contains an isomorphic replica $S_{0}^{\prime}$ of $S$, namely the set of all $\left(\lambda_{c}, \rho_{c}\right)$ with $c$ in $S$. If we identify $S_{0}^{\prime}$ with $S$, then $S^{\prime}$ is an extension of $S$ with the properties

$$
(\lambda, \rho) s=\lambda s, \quad s(\lambda, \rho)=s \rho .
$$

With any extension $\Sigma(0)$ of $S$ by $T$ we can associate a ramified homomorphism $A \rightarrow \bar{A}$ of $T^{*}$ into $S^{\prime}$, namely $\bar{A}=\left(\lambda_{A}, \rho_{A}\right)$, where $\lambda_{A} s=A \circ s$, $s \rho_{A}=s \circ A$. It ramifies within $S\left(=S_{0}^{\prime}\right)$ in the sense that $\bar{A} \bar{B} \in S$ if $A B=0$. Consequently, by Theorem 2 applied to $S^{\prime}$, to any $\Sigma(0)$ corresponds an extension $\Sigma^{\prime}(0)$ of $S^{\prime}$ by $T$ containing $\Sigma(0)$. Since $S^{\prime}$ contains a two-sided identity element, every extension of $S^{\prime}$ is obtained this way, by Theorem 2 . Thus, to construct all extensions $\Sigma$ of $S$ by $T$, we may construct all extensions $\Sigma^{\prime}$ of $S^{\prime}$ by $T$ by finding all ramified homomorphisms of $T$ into $S^{\prime}$ (at least one always exists) and then examine these to see which ones have the property that $\left.\bar{A} \bar{B} \in S=S_{0}^{\prime}\right)$ whenever $A B=0$ in $T$.

The Johns Hopkins University, BALTIMORE, MD. 